\title{
PREVALÊNCIA DO TRANSTORNO DE EVITAÇÃO ESCOLAR EM ADOLESCENTES ESCOLARES COM EXCESSO DE PESO DE CAMPINA GRANDE - PB
}

\section{PREVALENCE OF SCHOOL AVOIDANCE DISORDER IN SCHOOL ADOLESCENTS WITH EXCESS WEIGHT IN CAMPINA GRANDE - PB}

Lucenildo Laerty da Silva Sales ${ }^{1}$ Elayne Maria Cordeiro Costa ${ }^{2}$ José Ewayr Mariano de Araújo ${ }^{3}$ Carla Campos Muniz Medeiros ${ }^{4}$ Danielle Franklin de Carvalho ${ }^{5}$

RESUMO: Objetivo: Identificar a presença do transtorno de evitação escolar (EVE) em adolescentes regularmente matriculados no ensino médio de escolas públicas do município de Campina Grande - Paraíba. Metodologia: Estudo transversal, realizado com 131 adolescentes, com idades entre 15 e 19 anos de escolas públicas de Campina Grande - PB. Para avaliação do estado nutricional foi realizado o cálculo de índice de massa corporal (IMC) e para avaliação da presença ou ausência de do transtorno de evitação escolar foi utilizado o Questionário para Triagem de Ansiedade Infantil (QTAI-C). A análise estatística dos dados foi feita a partir do Statistical Packages for the Social Scientes - SPSS, versão 21.0. Para associação entre IMC, transtorno de evitação escolar e sexo foi realizado teste quiquadrado, considerando-se intervalo de confiança de 95\%. Resultados: Foi identificado a presença de algum transtorno de ansiedade em $32,1 \%$ dos pesquisados e, dentre esses, 20,6\%, apresentaram Evitação Escolar (EVE), o qual foi significativamente prevalente $(p<0,05)$ nos indivíduos eutróficos do sexo feminino $(30,3 \%)$. Ainda, $22,5 \%$ dos eutróficos apresentaram EVE, enquanto $18,3 \%$ dos casos apresentaram sobrepeso/obesidade. Considerações Finais: É evidente a

\footnotetext{
${ }^{1}$ Enfermeiro. Especialista em Gestão e Auditoria em Serviços de Saúde. Mestrando em Saúde Pública-PPGSP/UEPB.

2 Enfermeira. Especialista em Urgência, Emergência e UTI. Mestre em Saúde Pública PPGSP/UEPB.

${ }^{3}$ Farmacêutico. Especialista em Farmacologia clínica e atenção farmacêutica. Mestrando em Saúde Pública - PPGSP/UEPB.

${ }^{4}$ Médica. Professora do Programa de Pós-Graduação em Saúde Pública. Doutora em Saúde da Criança e Adolescente.

${ }^{5}$ Farmacêutica. Professora do Programa de Pós-Graduação em Saúde Pública. Doutora em Saúde da Criança e Adolescente.
} 
maior prevalência da evitação escolar no sexo feminino, o que se deve a predisposição ao desenvolvimento de transtornos de ansiedade, possivelmente em razão de aspectos sociais, genéticos e hormonais, sendo necessários estudos mais aprofundados nesse grupo. Além disso, não foi possível relacionar o estado nutricional e a prevalência de EVE.

Palavras chave: Transtornos Fóbicos. Obesidade. Saúde do Adolescente. Evasão Escolar.

ABSTRACT: Objective: To identify the presence of school avoidance disorder $(S A D)$ in adolescents regularly enrolled in public high schools in the city of Campina Grande - Paraiba. Methodology: Cross-sectional study, carried out with 131 adolescents, aged between 15 and 19 years, from public schools in Campina Grande - PB. The body mass index (BMI) was calculated to assess nutritional status and the Child Anxiety Screening Questionnaire (QTAI-C) was used to assess the presence or absence of school avoidance disorder. The statistical analysis of the data was made using the Statistical Packages for the Social Scientes - SPSS, version 21.0. For association between BMI, school avoidance disorder and sex, a chi-square test was performed, considering a 95\% confidence interval. Results: The presence of some anxiety disorder was identified in $32.1 \%$ of those surveyed and, among these, $20.6 \%$, had School Avoidance (SAD), which was significantly prevalent $(p<0.05)$ in eutrophic individuals female (30.3\%). In addition, $22.5 \%$ of eutrophic patients had EVE, while $18.3 \%$ of cases had overweight / obesity. Final Considerations: The higher prevalence of school avoidance in females is evident, which is due to the predisposition to the development of anxiety disorders, possibly due to social, genetic and hormonal aspects, requiring further studies in this group. In addition, it was not possible to relate the nutritional status and the prevalence of SAD.

Keywords: Phobic Disorders. Obesity. Adolescent Health. Student Dropouts. 


\section{INTRODUÇÃO}

Considera-se adolescência o período da vida humana compreendido entre as idades de 10 aos 19 anos, marcado por inúmeras mudanças biopsicossociais, sendo definido como um período de crises e conflitos, aumentando a susceptibilidade desses indivíduos ao desenvolvimento de diversos distúrbios psicológicos (GROLLI; WAGNER; DALBOSCO, 2019).

A obesidade pode ser definida como uma doença de etiologia multifatorial caracterizada pelo aumento excessivo da deposição de gordura corporal que pode trazer malefícios à saúde tanto física quanto mental (ABESO, 2016).

Segundo a Organização Mundial da Saúde (OMS), o número de crianças e adolescentes que apresentam excesso de peso e obesidade aumentou dez vezes nas últimas quatro décadas, caracterizando-se, assim, como um problema de saúde pública e uma epidemia global (WHO, 2017).

A obesidade, considerada como uma pandemia nos países mais pobres, afeta cerca de $60 \%$ dos indivíduos que vivem em países em desenvolvimento. Em países com maior PIB, os casos de obesidade estão presentes entre os residentes de áreas mais pobres e degradadas dos grandes centros e nas regiões rurais. (SMITH E SMITH, 2016; NG et. al. 2014; POPKIN et. al., 2012).

De acordo com Sánchez (2017), os adolescentes com sobrepeso/obesidade estão mais propensos a sofrer de problemas emocionais como ansiedade, baixa autoestima, depressão, descriminação e, inclusive, baixo rendimento acadêmico.

Além dos danos físicos e elevados custos de tratamento, o acúmulo de gordura pode causar alterações comportamentais, pois, pessoas com excesso são fáceis alvos de preconceito e discriminação, podendo ocasionar a manifestação de distúrbios psicossociais (SOUSA, et. al., 2012). Desse modo, pode-se entender que existe a possibilidade de que transtornos de ordem mental influenciem no acúmulo de gordura corporal. 
A ansiedade pode ser definida como um sentimento de perigo, que mostra há presença algo a ser temido, e pode manifestar-se de forma fisiológica e cognitiva. Dependendo de sua intensidade, é capaz de perturbar os indivíduos e inclusive causar sofrimento psíquico (OLIVEIRA; SISTO, 2002).

O ambiente escolar é um espaço de desenvolvimento e interações sociais benéficas ou não ao indivíduo, dentre elas estão as diversas formas de violência. $O$ bullying é uma dessas formas que tem sido amplamente praticado nas escolas e pode trazer inúmeras consequências negativas para a saúde mental do indivíduo que o sofre. (ZEQUINAO, 2016).

$\mathrm{Na}$ fase escolar, a ansiedade pode se mostrar aparente em razão de uma série de situações, caracterizando assim o transtorno de evitação escolar (EVE). O funcionamento social do estudante pode indicar sintomas de ansiedade, uma vez que as relações interpessoais entre os alunos requerem certa capacidade de adaptação. As situações avaliativas também podem produzir altos níveis de ansiedade, uma vez que a época de provas escolares acarreta no medo de fracassar e na redução do desempenho escolar. Desse modo, a ansiedade no contexto escolar pode se manifestar de diferentes maneiras, inclusive com problemas fisiológicos que afetam o funcionamento cognitivo (CUNHA, 2017).

O transtorno de evitação escolar pode ser definido como uma "condição clínica, ligada a um estado agudo de ansiedade relativa ao comparecimento à escola" (VELLOSO, 1973). Além do bullying, a evitação escolar também pode ser causada pela dificuldade de adaptação ao ambiente escolástico, e a pressão social de aprovação, principalmente durante o ensino médio. (GONZAGA; DA SILVA; ENUMO, 2017).

\section{OBJETIVO}

Identificar a prevalência de Transtorno da Evitação Escolar em adolescentes escolares matriculados no ensino médio de escolas públicas do município de Campina Grande - Paraíba. 


\section{METODOLOGIA}

Este é um estudo transversal, de natureza quantitativa, que foi realizado na cidade de Campina Grande - PB, em duas escolas de ensino médio integral, com maior prevalência de sobrepeso/obesidade (RAMOS et al, 2016).

Foram avaliados 131 adolescentes, sendo 71 eutróficos e 60 com sobrepeso/obesidade, na faixa etária entre 15 e 19 anos, matriculados nas duas primeiras séries do ensino médio. Como critérios de elegibilidade, estabeleceu-se que cursassem regularmente o ensino médio, não estivesse em tratamento ou tivessem histórico de transtornos de ansiedade e que não apresentassem limitação motora ou mental que impedisse sua participação na pesquisa. Estar cursando o terceiro ano do ensino médio foi o critério de exclusão do estudo, visto que essa população está naturalmente submetida a elevados níveis de ansiedade decorrente do momento acadêmico vivenciado.

As variáveis abordadas no estudo foram o estado nutricional, definido através do Índice de Massa Corporal (IMC), obtido através da razão entre o peso, em quilogramas, e o quadrado da estatura, em metros, e a presença ou ausência de sintomas de transtorno de ansiedade, mais especificamente do transtorno de evitação escolar (EVE), obtido através da aplicação do Questionário para Triagem de Ansiedade Infantil (QTAI-C), composto por 41 perguntas nas quais cada uma delas permite 3 opções de resposta, sendo contabilizadas as pontuações iguais ou superiores a 3 para os itens 2, 11, 17 e 36, que indicam EVE.

Através de uma reunião com todos os estudantes para explicação da pesquisa, realizamos a classificação nutricional através dos dados antropométricos inseridos no software Anthroplus $₫$. Os alunos com sobrepeso e obesidade que estavam dentro dos critérios de elegibilidade do estudo foram convidados a participar da pesquisa. Os indivíduos que aceitaram, foram orientados sobre todas as etapas posteriores do estudo e após isso, assinaram o Termo de Assentimento 
Livre e Esclarecido (TALE) e seus responsáveis, o Termo de Consentimento Livre e Esclarecido (TCLE), em duas vias.

Os dados coletados foram duplamente digitados e, posteriormente, sujeitados à validação pelo Validate do Epi Info 6.04. Para a realização do processo de análises estatísticas o programa foi utilizado o SPSS, versão 21.0.

Realizou-se a análise descritiva das variáveis estudadas através de frequência absoluta e relativa. Para avaliação da associação entre o estado nutricional e do sexo com o transtorno de evitação escolar, foi realizado teste quiquadrado. Para todas as análises foi considerado um intervalo de confiança de $95 \%$.

Esse estudo foi aprovado no comitê de ética da Universidade Estadual da Paraíba sob o CAAE $n^{\circ}$ 0077.0.133.000-12, em conformidade com a Resolução $n^{\circ}$ 466/12 do Conselho Nacional de Saúde.

\section{RESULTADOS E DISCUSSÃO}

Dos 131 estudantes entrevistados, $42 \%$ do sexo masculino e $58 \%$ do sexo feminino. Dentre estes $54,2 \%$ pertenciam ao grupo dos eutróficos e $45,8 \%$ ao grupo dos que apresentavam sobrepeso ou obesidade.

Após a análise dos dados, apurou-se que 32,1\% dos pesquisados possuem algum tipo de Transtorno de Ansiedade (TA). Desses, a Evasão Escolar (EVE), representou $20,6 \%$ dos casos.

Corroborando a Kinrys e Wygante (2005) foi evidenciada uma maior prevalência da EVE no sexo feminino (30,3\%), que no sexo masculino (7,3\%). Além do mais, Laranjeira et al. (2014) afirma que os indivíduos do sexo feminino são as que mais sofrem com os diversos tipos de violência presente nas escolas, podendo esta razão explicar a maior prevalência da evitação escolar nesta população. 
Tabela 1 - Distribuição do Estado Nutricional, Transtornos de Ansiedade e Evitação Escolar em 131 adolescentes escolares de acordo com o sexo. Campina Grande PB, 2016.

\begin{tabular}{lcccc}
\hline & \multicolumn{2}{c}{ SEXO } & & \\
& Masculino & Feminino & RP (IC 95\%) & p \\
\hline VARIÁVEIS & $\mathbf{n ~ ( \% )}$ & $\mathbf{n ~ ( \% )}$ & & \\
Estado Nutricional & & & & \\
Eutroficos & $32(58,2)$ & $39(51,3)$ & $0,758(0,376-1,525)$ & 0,436 \\
Sobrepeso/ Obesidade & $23(41,8)$ & $37(48,7)$ & & \\
Transtornos & de & & & \\
Ansiedade (TA) & & & & \\
Presença & $11(20,0)$ & $31(40,8)$ & $0,363(0,162-0,811)$ & 0,012 \\
Ausência & $44(80,0)$ & $45(59,2)$ & & \\
Evitação Escolar (EVE) & $4(7,3)$ & $23(30,3)$ & & \\
Presença Ausência & $51(92,7)$ & $53(69,7)$ & $0,181(0,058-0,559)$ & 0,001 \\
\hline
\end{tabular}

Já quando confrontado com o estado nutricional, a EVE esteve presente em $22,5 \%$ dos analisados eutróficos e em 18,3\% dos analisados com sobrepeso ou obesidade, o que pode estar relacionado com a grande prática de bullying contra pessoas com sobrepeso e obesidade, principalmente escolares, que os levam a buscar o isolamento, como alternativa à agressão (ALEXIUS, 2016).

Tabela 2 - Distribuição dos Transtornos de Ansiedade e de Evitação Escolar em 131 adolescentes escolares de acordo com o estado nutricional. Campina Grande - PB, 2016.

\begin{tabular}{lcccc}
\hline \multicolumn{5}{c}{ ESTADO NUTRICIONAL } \\
& Eutrófico $\mathbf{n}(\%)$ & $\begin{array}{c}\text { Sobrepeso I } \\
\text { Obesidade n (\%) }\end{array}$ & RP (IC 95\%) & p \\
\hline VARIÁVEIS & Transtornos de Ansiedade (TA) & \\
Presença & $23(32,4)$ & $19(31,7)$ & 0,967 & 0,929 \\
Ausência & $48(67,6)$ & $41(68,3)$ & $(0,463-2.021)$ & \\
Presença & Evitação Escolar (EVE) & & \\
Ausência & $16(22,5)$ & $11(18,3)$ & 0,772 & 0,554 \\
\hline
\end{tabular}


Para Figueiroa, 2015, indivíduos com excesso de peso, principalmente mulheres, possuem maior prevalência TAs. Por outro lado, altos níveis de ansiedade podem explicar a etiologia da obesidade. Em suma, pouco se compreende a origem dos fatores que elevam as chances de mulheres desenvolverem um transtorno de ansiedade no decorrer da vida.

Os hormônios femininos têm sua contribuição no que concerne a frequência, gravidade e curso dos TAs. Ainda, os ciclos menstruais, a resposta às situações de medo e perigo desencadeiam maiores respostas emocionais quando comparado ao sexo oposto. Evidências científicas sugerem que isso pode acontecer pelo fato do córtex anterior do giro do cíngulo ser anatomicamente maior e mais ativa nas mulheres, e isso causa respostas emocionais e racionais mais intensas no sexo feminino, elevando as tensões e os níveis de ansiedade (KINRYS, 2005).

Por outro lado, esses resultados podem ser explicados por questões apontam a relevância do papel da mulher e os aspectos emocionais envolvidos nas relações familiares e sociais. Diversas mudanças ocorridas com o passar dos anos, decorrentes dos movimentos sociais, alteraram a posição da mulher na sociedade, especialmente a adolescente, tornando-as tão permissíveis quanto os homens. Assim, ambos contribuem para a imposição de novos modelos de relação social e familiar contemporâneos, reduzindo as perspectivas ocasionam desgaste emocional e acarreta em acúmulo de tensões que convergem para o desenvolvimento dos TAs e sua prevalência nessa população. (BORSA; NUNES, 2017).

No entanto, embora presente em adolescentes com obesidade ou sobrepeso, ainda não é possível associar o estado nutricional com a evitação escolar, uma vez que os dados obtidos pelo presente estudo não foram suficientes para estabelecer tal ligação. Além do mais, não é possível encontrar na literatura aparato para designar essa associação.

Baseando-se nas informações coletadas, a prevalência dos transtornos ansiosos nos adolescentes e sua vulnerabilidade biológica e psicossocial exigem manejo habilidoso para assegurar suporte terapêutico de qualidade. Além disso, as particularidades inerentes ao adolescente podem explicar a falta de relação entre o estado nutricional e os transtornos de ansiedade, uma vez que esse período traz 
características muito específicas que podem afetar as análises. (CRUZ, 2017; DA SILVA FILHO; SILVA, 2017).

\section{CONSIDERAÇÕES FINAIS}

Através da análise dos dados, pudemos perceber que os transtornos de ansiedade, incluindo o transtorno de evitação escolar, estão presentes em adolescentes escolares independente do estado nutricional, porém mais prevalentes nos indivíduos do sexo feminino, o que revela a necessidade de uma investigação mais amplas a respeito da prevalência nesse grupo específico. Isso, supostamente, se deve tanto à fatores genéticos, quanto por influência hormonal.

Assim, faz-se necessário outros estudos que apontem causalidade e investiguem profundamente essa associação.

\section{REFERÊNCIAS BIBLIOGRÁFICAS}

ALEXIUS, Sílvia Letícia et al. Sobrepeso e obesidade e associação com Bullying em escolares do município de Florianópolis, Santa Catarina, Brasil. 2016.

BORSA, Juliane Callegaro; NUNES, Maria Lucia Tiellet. Aspectos psicossociais da parentalidade: o papel de homens e mulheres na família nuclear. Psicologia Argumento, v. 29, n. 64, 2017.

CRUZ, Danilly Rafaelly Martins; BORGES, Lucivanda Cavalcante. A queixa escolar: reflexões sobre o atendimento psicológico. Psicologia Argumento, v. 31, n. 72, 2017.

DA SILVA FILHO, Orli Carvalho; DA SILVA, Mariana Pereira. Transtornos de ansiedade em adolescentes: considerações para a pediatria e hebiatria. Adolescencia e Saude, v. 10, n. 3, p. 31-41, 2013.

DE OBESIDADE, Diretrizes Brasileiras. Associação Brasileira para o Estudo da Obesidade e da Síndrome Metabólica. (2016). 2016.

FIGUEROA, Ana et al. Ansiedade de separação. Tratado de Saúde Mental da Infância e Adolescência da IACAPAP. 2015.

GONZAGA, Luiz Ricardo Vieira; DA SILVA, Andressa Melina Becker; ENUMO, Sônia Regina Fiorim. Ansiedade de provas em estudantes do Ensino Médio. Psicologia Argumento, v. 34, n. 84, 2017.

GROLLI, Verônica; WAGNER, Marcia Fortes; DALBOSCO, Simone Nenê Portela. Sintomas 
depressivos e de ansiedade em adolescentes do ensino médio. Revista de Psicologia da IMED, v. 9 , n. 1, p. 87-103, 2017.

KINRYS, Gustavo; WYGANT, Lisa E. Transtornos de ansiedade em mulheres: gênero influencia o tratamento?. Brazilian Journal of Psychiatry, v. 27, p. s43-s50, 2005.

KINRYS, Gustavo; WYGANT, Lisa E. Transtornos de ansiedade em mulheres: gênero influência o tratamento?. Revista Brasileira de Psiquiatria, 2005.

LARANJEIRA, Ronaldo et al. II levantamento nacional de álcool e drogas (LENAD)-2012. São Paulo: Instituto Nacional de Ciência e Tecnologia para Políticas Públicas de Álcool e Outras Drogas (INPAD), UNIFESP, 2014.

NG, M. et al. Global, regional, and national prevalence of overweight and obesity in children and adults during 1980-2013: a systematic analysis for the Global Burden of Disease Study 2013The Lancet, Volume 384 , Issue 9945,766 - 781, 2014. 10.

OLIVEIRA, Sandra Maria da Silva Sales; SISTO, Fermino Fernandes. Estudo para uma escala de ansiedade escolar para crianças. Psicologia Escolar e Educacional, v. 6, n. 1, p. 57- 66, 2002.

POPKIN, B.M. ADAIR, L.S. NG, S.W. Global nutrition transition and the pandemic of obesity in developing countries. Nutrition Reviews. Jan;70 (1):3-21, 2012.

RAMOS, Thacira DA et al. Assessment of the carotid artery intima-media complex through ultrasonography and the relationship with Pathobiological Determinants of Atherosclerosis in Youth. Cardiology in the Young, v. 26, n. 7, p. 1333-1342, 2016.

SÁNCHEZ, Gustavo Gutiérrez et al. Ansiedad como factor asociado a la obesidad en adolescentes/Anxiety as a factor associated with obesity in adolescents/Ansiedade como fator associado à obesidade em adolescentes. JOURNAL HEALTH NPEPS, v. 2, n. 2, p. 302-314, 2017.

SILVA, Glauciene dos R.; CRUZ, Nilcemar R.; COELHO, Ering Júnior B. Perfil nutricional, consumo alimentar e prevalência de sintomas de anorexia e bulimia nervosa em adolescentes de uma escola da rede pública no município de Ipatinga, MG. Revista Digital de Nutrição, v. 2, n. 3, p. 1-15, 2008.

SMITH, K .B. M.S, SMITH. Obesity Statistics Primary Care: Clinics in Office Practice, Volume 43, Issue $1,121-135,2016$.

SOUSA ALMEIDA, Sebastião; PEROCO ZANATTA, Daniela; FARIA REZENDE, Fabiana. Imagem corporal, ansiedade e depressão em pacientes obesos submetidos à cirurgia bariátrica. Estudos de Psicologia, v. 17, n. 1, 2012.

VELLOSO, Elisa Dias. Aspectos da fobia escolar. Arquivos Brasileiros de Psicologia Aplicada, v. 25, n. 3 , p. 39-50, 1973.

WORLD HEALTH ORGANIZATION - WHO. Obesity and Overweight. Important Facts. Oct. 2017. Disponível em: <http://www.who.int/en/news-room/facts-sheets/details/obesity- andoverweight> Acesso em: 12. Fev. 2019.

ZEQUINÃO, Marcela Almeida et al. Bullying escolar: um fenômeno multifacetado. Educação e Pesquisa, v. 42, n. 1, p. 181-198, 2016. 